\title{
Multi-Modality Imaging of Craniofacial Fibrous Dysplasia with Orbital Complications and Histopathology Correlation-A case report and short review of literature
}

\author{
Chithra Ram MD ${ }^{*}$, Hayley Moss ${ }^{2}$, Merryl Terry MD³ . Manasaa Kannan ${ }^{4}$, Richard Sherry MD \\ ${ }^{1}$ Neuroradiologist, Assistant Professor, Department of Radiology, University of Louisville, Kentucky, USA. \\ ${ }^{2}$ Medical student, University of Louisville, Kentucky, USA. \\ ${ }^{3}$ Resident, Department of Pathology, University of Louisville, Kentucky, USA. \\ ${ }^{4}$ Medical student, University of Louisville, Kentucky, USA. \\ ${ }^{5}$ Neuroradiologist, RG Sherry MD PLLC, Watertown, New York, USA.
}

*Corresponding Author: Chithra Ram, Neuroradiologist, Assistant Professor, Department of Radiology, University of Louisville, Kentucky, USA.

Received date: June 08, 2021; Accepted date: June 22, 2021; Published date: June 25, 2021

Citation: C Ram, H Moss, M Terry, M Kannan, R Sherry. (2021) Multi-Modality Imaging of Craniofacial Fibrous Dysplasia with Orbital Complications and Histopathology Correlation-A case report and short review of literature. International Journal of Clinical Case Reports and Reviews. 7(3); DOI:10.31579/2690-4861/144

Copyright: (c) 2021 Chithra Ram, This is an open-access article distributed under the terms of the Creative Commons Attribution License, which permits unrestricted use, distribution, and reproduction in any medium, provided the original author and source are credited.

\begin{abstract}
Craniofacial fibrous dysplasia [CF-FD] with orbital complications is a known but rare entity. This is a multi-modality imaging Case Report of extensive CF-FD in a 31-year-old male with right eye pain, swelling, and redness, along with histopathology correlation. In this patient, the CT scan demonstrates the classic ground glass bony appearance in great detail and helps with the diagnosis of FD, while excluding other bony pathology. The patient's corroborative MRI brain with and without contrast and MRI brain perfusion images are presented to further characterize this pathology along with its orbital and ocular complications. Given the significant mass-effect on the ocular structures, the patient underwent orbital surgery with removal of as much of the lesion as possible. On macroscopic pathology evaluation, the affected bone was rubbery and gritty when sectioned. Microscopically, remnant fragments of woven bone of various size and shapes were seen with lack of an osteoblast rim. The bony fragments had a characteristic curvilinear, trabecular, and/or branching pattern. Post-surgical imaging demonstrated improvement in the mass-effect on orbital structures and proptosis, along with residual bony lesion.
\end{abstract}

Key Words: craniofacial fibrous dysplasia; CT of craniofacial fibrous dysplasia; MRI of craniofacial fibrous dysplasia; MRI perfusion of fibrous dysplasia; fibrous dysplasia imaging; histopathology of fibrous dysplasia

\section{Introduction}

Fibrous dysplasia [FD] forms $7 \%$ of benign bone tumors. FD usually presents within the first two decades of life. However, our patient was over 30 years of age. It has no gender predilection. Approximately $85 \%$ of cases of fibrous dysplasia are associated with activated missense mutations in GNAS gene with stimulation of adenylyl cyclase and overexpression of cAMP. FD can be monostotic or polyostotic. Monostotic lesions are most common. A very small percent of these lesions can be large enough to cause complications. E.g. involvement of a large portion of the orbital walls with mechanical compression on the orbital structures resulting in related signs and symptoms, etc. Craniofacial involvement may occur both as monostotic or polyostotic fibrous dysplasia. Occasionally it is seen in McCune-Albright syndrome.

\section{Case Report}

A 31-year-old male presented to the emergency department with right eye pain, swelling, and redness that began the prior morning. There was no history of trauma and he denied any visual disturbances. Prior medical history was significant for hypertension. Vital signs were notable for a blood pressure of 179/92. Physical exam showed a large solid lesion in the right fronto-orbital region and proptosis. Neurologic examination showed decreased sensation to light touch on the right side of the face. 
The rest of the body including the extremities were unremarkable. CT and MRI were obtained to evaluate the right fronto-orbital lesion.

\section{Methods}

CT head without contrast was performed in a Somatom Definition Edge Siemens scanner [Siemens Medical Systems, USA]. Standard 1 mm axial CT soft tissue and bone detail algorithm images were obtained followed by reconstructed images in coronal and sagittal planes.

MRI of the brain was performed on a 3T Siemens Spectra scanner [Siemens Medical Systems, Malvern, Pennsylvania, USA] using a standard head coil. Standard $4 \mathrm{~mm}$ thick axial T1, axial T2, fat suppressed axial T2 FLAIR, axial DWI and ADC brain pulse sequences were acquired without contrast. After intravenous administration of $20 \mathrm{ml}$ of
Multihance, perfusion imaging was performed along with axial and coronal post contrast $\mathrm{T} 1 \mathrm{~W}$ sequences.

\section{Imaging Findings}

CT examination of the brain showed a large, expansile, sclerotic lesion in the right orbital roof and sphenoid bone with infiltration of the ethmoid sinuses characterized by benign appearing margins and a uniform classic "ground-glass" appearing matrix (Fig.1a,b). The bony contour of the right supraorbital region was smoothly expanded. The expansile right orbital bony lesion compromised the total volume of the right orbit and caused severe proptosis (Fig. 1c, d, e), mass-effect on the extra-ocular muscles particularly lateral rectus and probable optic nerve compression due to the narrowed optic canal.

\section{IMAGING FIGURES: Craniofacial Fibrous dysplasia}
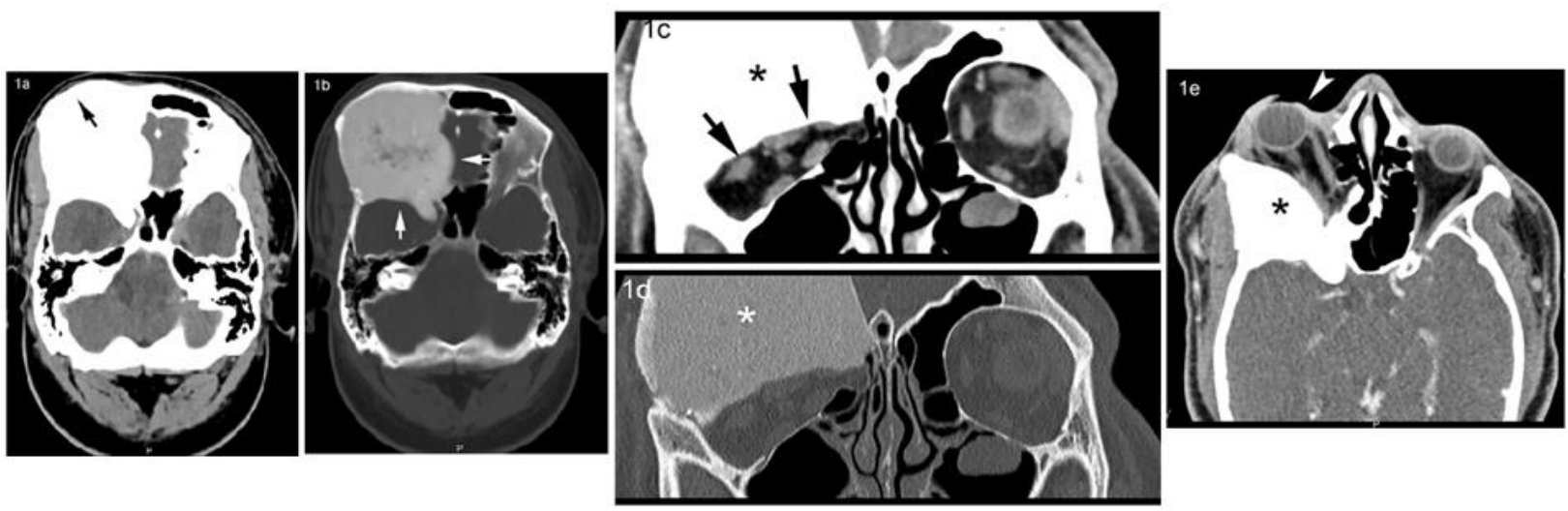

Figure 1: CT brain. Axial non-contrast brain and bone window images show smooth, expansile deformity of the right supraorbital skull (black arrow Figure 1a). There is extensive involvement of the right orbital roof with the lesion showing the characteristic ground-glass appearance of fibrous dysplasia. The thinned and expanded frontal bone shows endosteal scalloping while the sphenoid and ethmoid interfaces lack a distinct cortex (white arrows Figure $1 b$ ). Coronal soft tissue and bone window images demonstrate the markedly thickened right orbital roof with typical appearance of FD (asterisk Figure 1c,d) and the severe encroachment on the orbit (arrows Figure 1c).Marked involvement of the lateral wall of the right orbit is apparent (asterisk Figure 1e) with significant orbital volume loss and severe proptosis (arrowhead Figure le).

The MRI appearance was in keeping with CT diagnosis of FD. It showed heterogeneous T1 hypointensity and mild diffuse enhancement after contrast administration (Fig. 2). Additional MRI perfusion imaging (Fig. 3a) confirmed that this was not a vascular lesion and helped exclude vascular lesions such as metastasis or meningioma. DWI sequences revealed no restricted diffusion (Fig. 3b, c). Based on the imaging findings and clinical presentation the lesion was felt to be consistent with CF-FD. The patient underwent subtotal resection of the large skull lesion with decompression of the orbit, optic nerve, and superior orbital fissure using microsurgical technique and intraoperative neuronavigation. Thin layers of the lesion were unable to be resected due to their close proximity to the frontal and ethmoid sinuses. Subsequent cranioplasty with titanium mesh was used to create the orbital rim (Fig. 4). Post-operatively he developed moderate vision loss in the right eye. Follow-up postoperative imaging showed no expansion of the fibro-osseous lesion. His most recent 7 years post-op imaging shows stable and unchanged remnants of the fibrous dysplasia and proptosis. No other intervention since initial craniotomy has been necessary. 

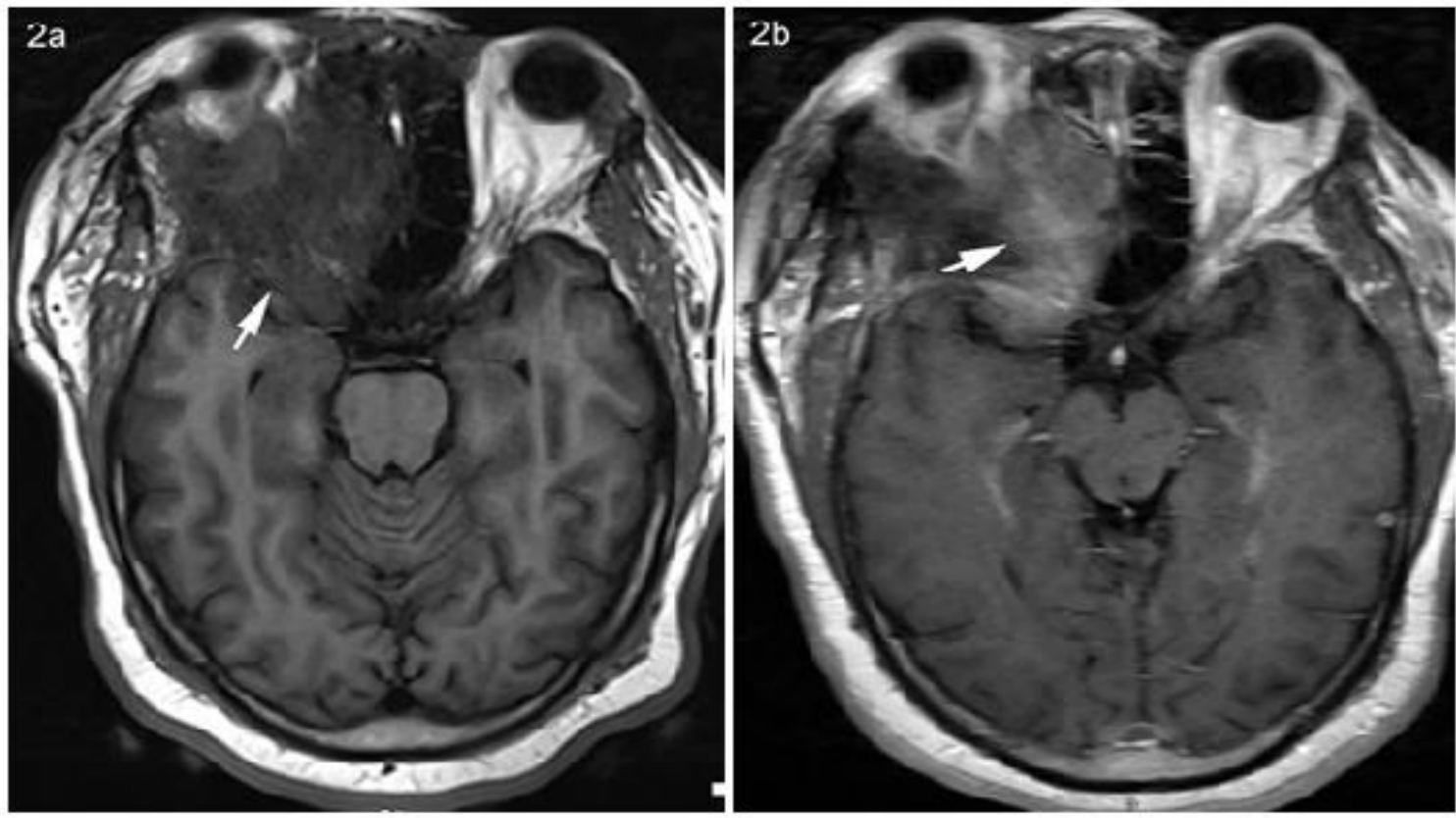

Figure 2: 3T Siemens Spectra scanner MRI Brain images with and without contrast. Axial non-contrast T1 image shows the extensive involvement of the right orbital roof by a slightly heterogeneous hypointense lesion (arrow Figure 2a). The corresponding axial post contrast T1 image demonstrates mild diffuse enhancement within the lesion (arrow Figure 2b), which can be a feature of FD.
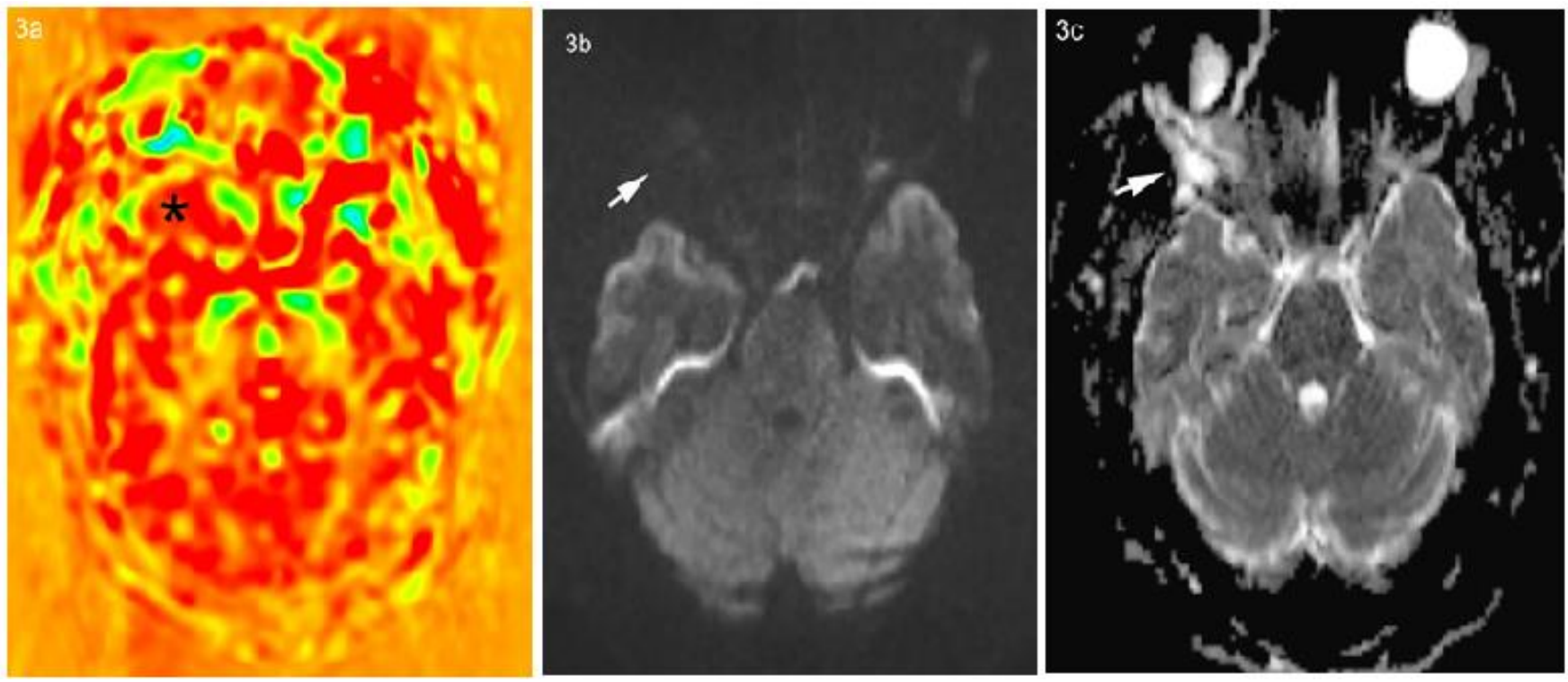

Figure 3: 3T Siemens Spectra scanner MRI brain perfusion image with contrast. There is no increased perfusion demonstrated in the right orbital lesion on MRI perfusion imaging (asterisk Figure 3a). Diffusion weighted sequences show no restricted diffusion in the lesion on DWI and ADC map images (arrows Figure 3b, 3c). 

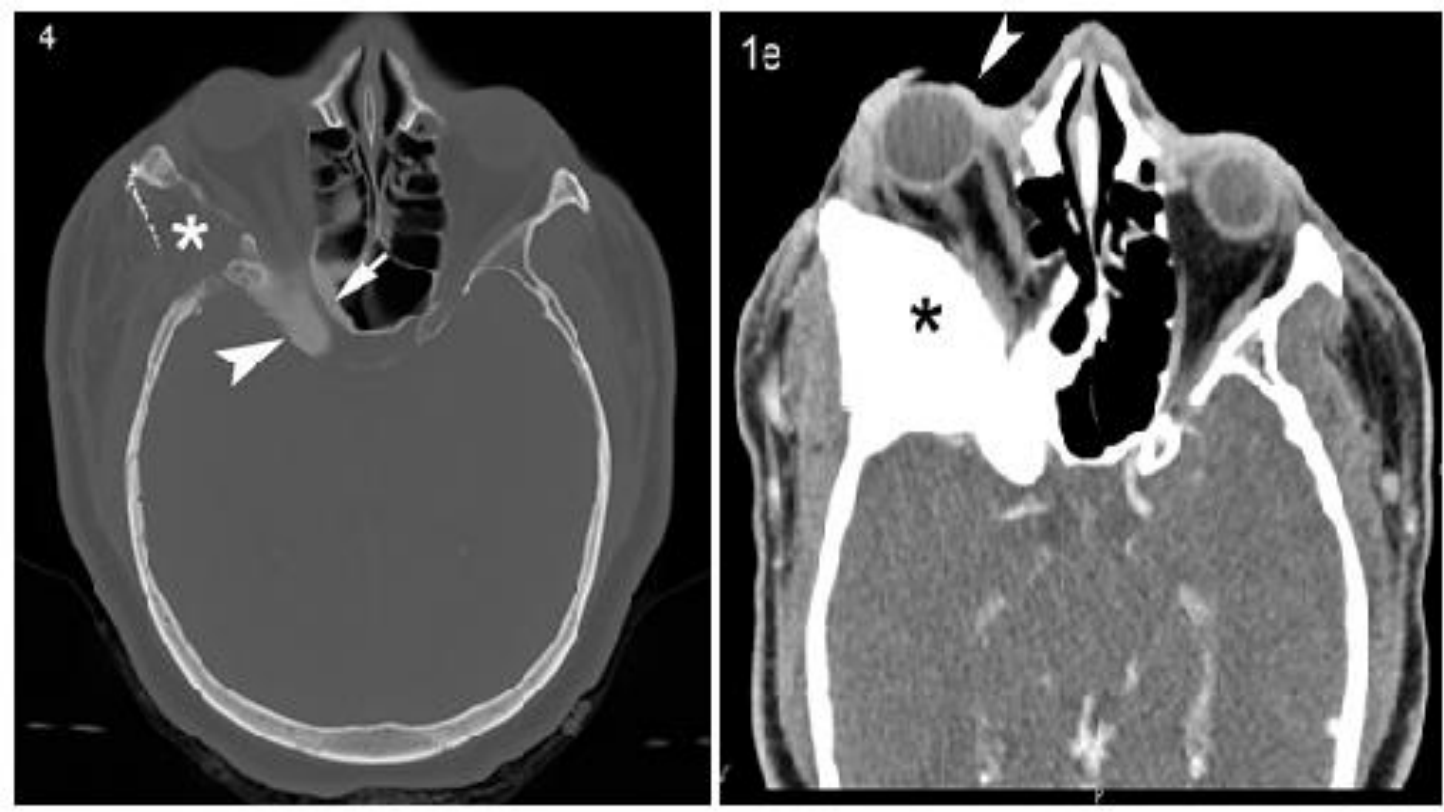

Figure 4: Axial CT bone window image [Figure 4] through the level of the orbits post-surgery shows partial resection of the lesion involving the right lateral orbital wall (asterisk) with decompression of the orbit and improvement of proptosis. Note FD involving the anterior clinoid process (arrowhead) and lateral wall of the sphenoid sinus with narrowing of the optic canal (arrow). The pre-op soft tissue window image through the orbit [Figure 1e] is again given to compare it with the post-op image [Figure 4], to see the extent of lesion removal and the resultant improvement of mass effect on the orbital structures.

\section{Histopathology Findings}

In the $4 \mathrm{X}$ low power hematoxylin and eosin (H\&E) stained slide of the specimen [Fig.5], a combination of fibrous appearing tissue and scattered bony trabeculae were well seen. The $10 \mathrm{X}$ mid power H\&E stained slide [Fig.6] demonstrated areas of hemorrhage associated with multinucleated osteoclast-type giant cells. Matrix resembling cementum was found in this craniofacial lesion. 20X High power H\&E stained slide [Fig.7] demonstrated moderately cellular stroma with storiform [whirling] and spindle shaped cells. Fibrous dysplasia was diagnosed based on the moderately cellular spindle cells arranged in storiform (cartwheel or whirling) pattern with low mitotic count amidst the woven, discontinuous bone trabeculae.

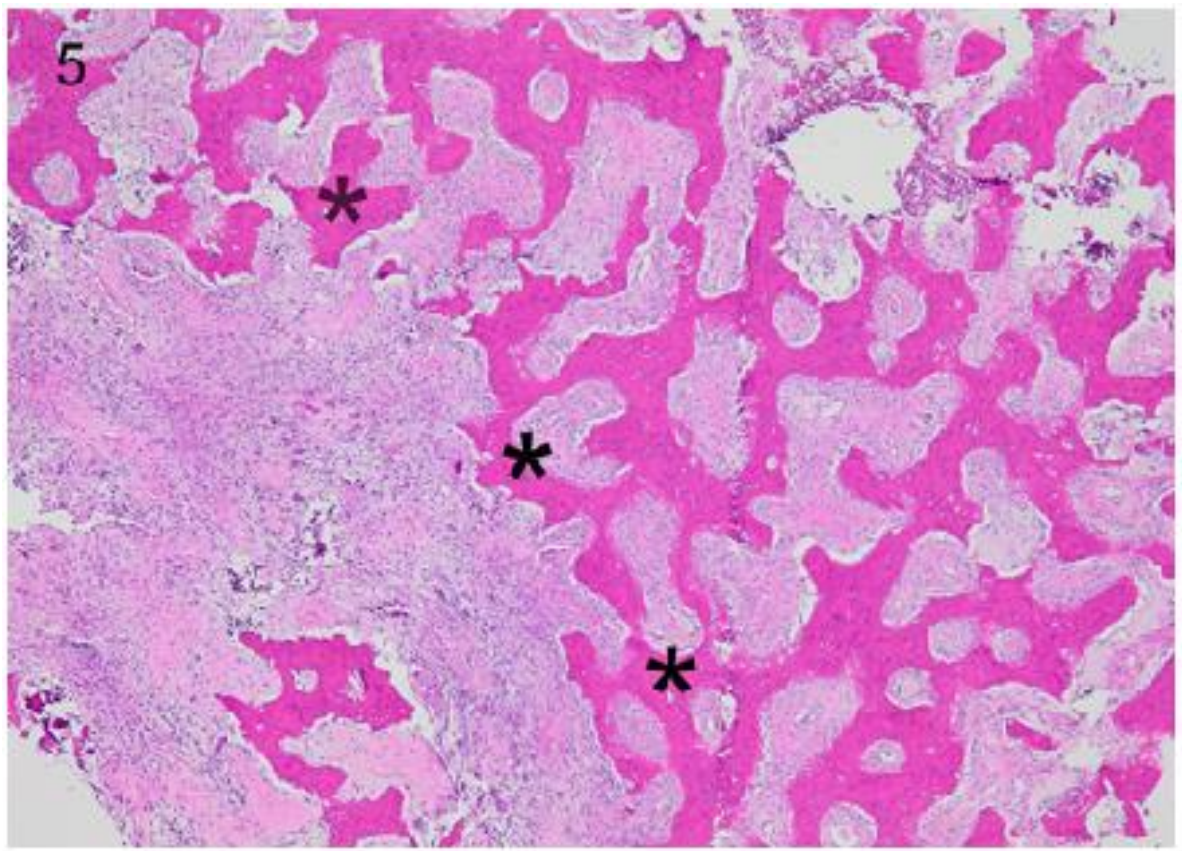

Figure 5: At low power magnification H\&E-stained section (4X magnification). Irregularly-shaped bony trabeculae (asterisks) with surrounding moderately cellular fibrous tissue, characteristic of fibrous dysplasia. 


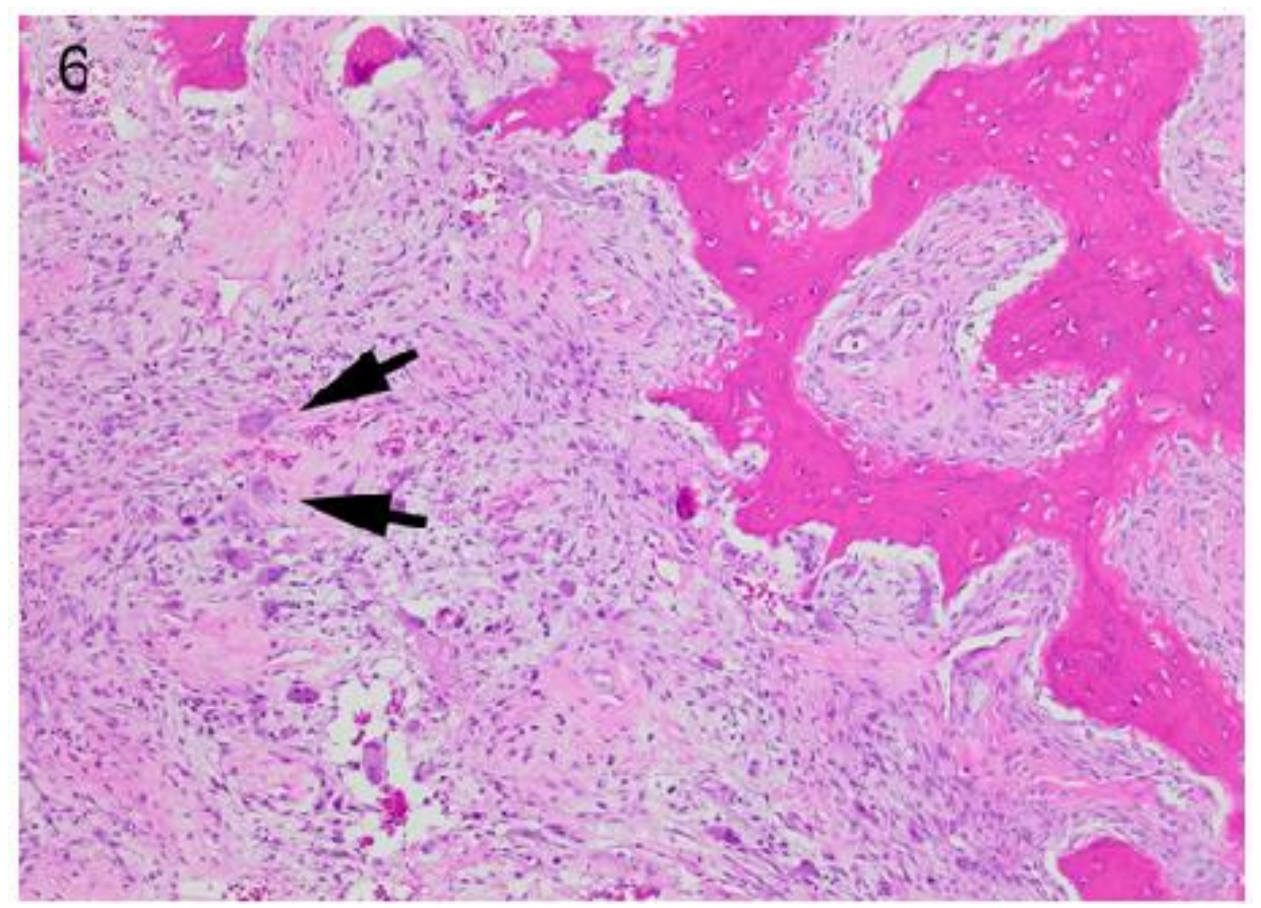

Figure 6: At mid power magnification (10X, H\&E stained section). Areas of stromal collagen deposition as well as scattered multinucleated cells (arrows) are visible. Note the absence of osteoblastic rim surrounding bony trabeculae. The boney islands are woven, rather than lamellar.

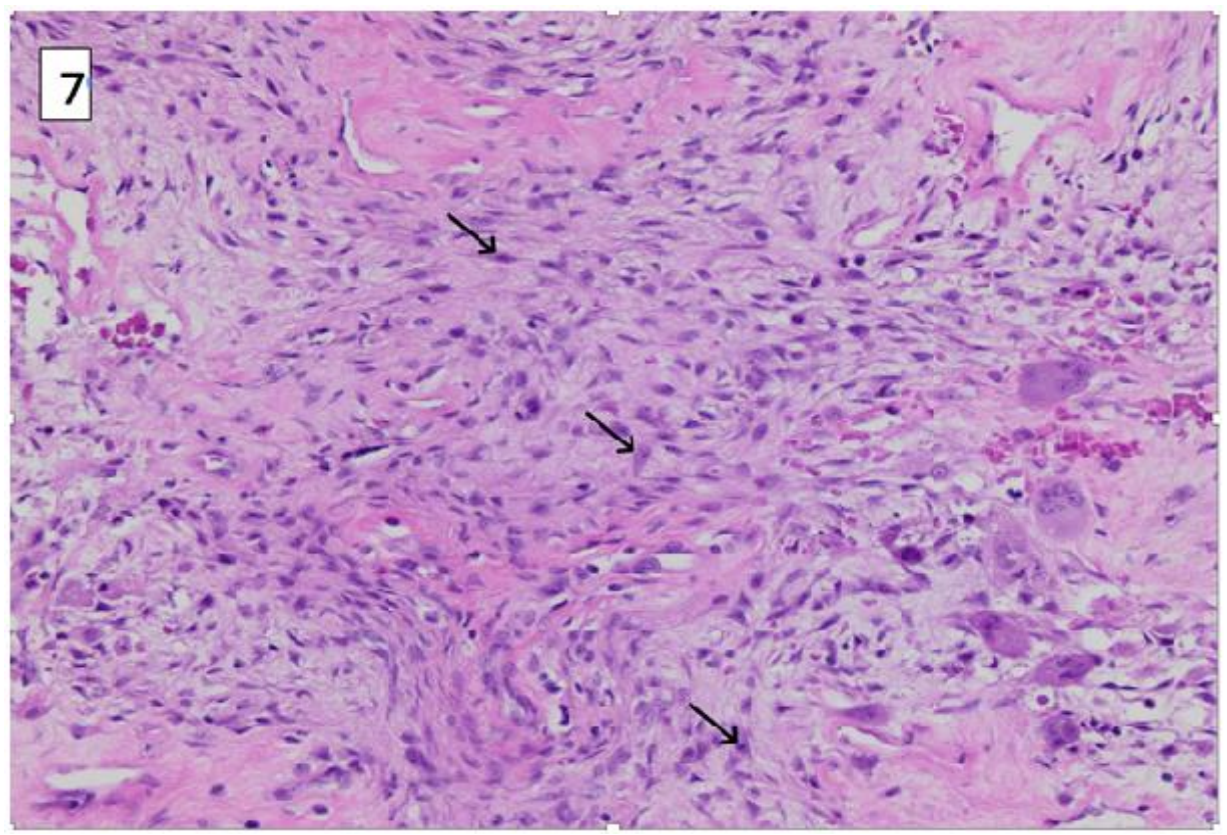

Figure 7: At High power H\&E stained section (20X magnification) of the stromal component. The stromal cells (arrows), primarily fibroblasts, are small, bland, spindled, or stellate. No cytologic atypia is seen. Scattered small vessels are also present.

\section{Discussion}

\section{Introduction}

Fibrous dysplasia [FD] is a well-known benign bony entity. It is a developmental disorder that results from a sporadic mutation that replaces bone with fibrous tissue, resulting in structural instability [1]. FD can be monostotic [involvement of one site] or polyostotic [involvement of 2 or more sites], with monostotic lesions being most common [2,3]. Craniofacial involvement in FD can be monostotic in $10-25 \%$ of monostotic cases or polyostotic in up to $50 \%$ of polyostotic cases. FD can also exist in conjunction with endocrinopathies (e.g. McCune-Albright Syndrome) [4]. Polyostotic FD is more commonly associated with symptomatic disease and deformity [5]. It's a non-hereditary disorder with expansile lesion(s) caused by a defect in osteoblastic differentiation and maturation. It contains a mixture of fibrous tissue and woven bone, giving rise to the characteristic ground glass appearance. Sarcomatous dedifferentiation can be rarely seen in polyostotic FD with prior radiation therapy. 
McCune-Albright Syndrome has a clinical triad of polyostotic FD, hyperfunctioning endocrinopathies and café au lait spots. It has a female preponderance [7]. Mazabraud Syndrome is polyostotic FD with intramuscular myxoma. It has increased risk of malignant transformation. Cherubism is familial FD involving bilateral jaw [8].

\section{Clinical Presentation}

FD is often asymptomatic and diagnosed incidentally as a wellcircumscribed lesion, especially with monostotic disease. Clinical presentation often depends on location and number of lesions. Ribs, femurs, and craniofacial bones are most commonly affected [3,6]. Craniofacial FD presents with asymmetric facial enlargement due to the affected bone, proptosis, atypical facial pain or numbness, headache and cranial neuropathy (diplopia, hearing loss, blindness) [9]. Progression from monostotic to polyostotic disease has not been documented [1]. Craniofacial bones are most commonly implicated in monostotic FD, while the femur is commonly affected in polyostotic FD. Polyostotic disease occurs in a younger age with limp, leg pain, or pathologic fractures. The lesions are commonly unilateral and are located in the same extremity when the appendicular skeleton is affected [8]. Fractures typically occur at the proximal femur with possible "shepherd's crook" deformity. If the spine is affected, then scoliosis can occur [3,6]. Patients with café-au-lait spots and precocious puberty with FD should be considered for a diagnosis of McCune-Albright syndrome [10]. Association with aneurysmal bone cysts has also been described [11].

\section{Imaging Findings}

Commonly discovered as an incidental finding, lesions of fibrous dysplasia are characterized into three types [12]. Pagetoid lesions are most common, followed by sclerotic and then cystic lesions [7]. In craniofacial FD, more than 1 bone involvement is often seen. Maxilla, orbit, and frontal bones are commonly involved, while ethmoids and sphenoids are rarely involved. CT is most sensitive and specific. CT and MR help define local extent, while bone scintigraphy scan identifies additional lesions [10].

Radiographs: FD is a smooth, well circumscribed, homogeneous ground glass lesion with well-defined borders. Endosteal scalloping and cortical thinning is noted due to the expansive nature of the lesion. It may be sclerotic or lucent (cystic). Sclerotic lesions are homogenously dense. Cystic lesions can show a "rind" sign, where a thick layer of sclerotic reactive bone surrounds a lucent lesion $[3,7,14]$. This sign is commonly seen in proximal femur.

CT: Radiolucent ground-glass matrix in an expansile bony lesion involving the medulla, is diagnostic especially in pagetoid lesions. Smooth cortical contour is typically maintained. Endosteal scalloping may also be present [13].

MRI: Decreased T2W signal is noted throughout the lesion if it's pagetoid or solid type and only in rim if it's of the cystic type. Increased pathological activity can result in fewer bony trabeculae and hence result in increased T2W signal [3,7]. Decreased T1W signal is usually seen. Enhancement is variable. It can be peripheral, diffuse or non-enhancing.

Bone scan: It's nonspecific with variable radionuclide uptake in perfusion and delayed phases. It's sensitive but not specific in identifying skeletal lesions in polyostotic FD.

PET: FD shows variable uptake on FDG [Fluoro-deoxy glucose] PET. Correlation with CT helps differentiate it from metastasis.

\section{Histopathology}

The histopathologic features of CF-FD consists primarily of normal bone that is replaced and distorted by bland fibrous tissue [15]. Grossly, the affected bone is rubbery and gritty when sectioned [15]. Microscopically, remnant fragments of woven bone of various size and shapes are seen. These bony fragments characteristically lack an osteoblast rim, and may seamlessly interface with adjacent cancellous bone or cortex $[15,16]$. As a result, there may be no clear demarcation between lesion and adjacent normal bone on imaging [17]. The bony fragments are curvilinear, trabecular, and/or branching, which gives a characteristic "Chinese letter" or "alphabet soup" appearance [15]. This manifests as diffusely distributed opacities on imaging. The bone fragments are typically trabecular or woven, although lamellar bone is occasionally present if the lesion has been present for many years [15-17]. The fibrous tissue is relatively cellular and contains spindled or stellate fibroblasts with associated collagen. Mitotic figures are scarce or absent [15]. Cases with abundant cartilage, named fibrocartilaginous dysplasia, have been described [15]. Synonyms that are no longer recommended for use include osteitis fibrosa, fibrous osteoma, and unilateral von Recklinghausen disease. Approximately $85 \%$ of cases of fibrous dysplasia are associated with activated missense mutations in GNAS gene with stimulation of adenyl cyclase and over-expression of cAMP $[15,18]$.

\section{Prognosis and Treatment}

Prognosis is good. Many cases spontaneously resolve with aging as the lesions become inactive after puberty, and the condition is considered benign. Asymptomatic cases can be managed with patient education and clinical observation [19]. The maintenance of bone density is important during management, especially for prevention of fractures [1]. Malignant transformation can rarely occur and is more common in polyostotic rather than monostotic FD [7]. No treatment methods are currently available to slow or alter the disease course. Bisphosphonates can control bone pain [19]. Polyostotic patients should be carefully monitored to optimize function and ambulation as they can result in pathological fractures, which may affect mobility. Surgery with grafting is an option to correct deformities or otherwise symptomatic lesions and large monostotic lesions can be resected $[3,19]$. Aggressive resection is reserved for visual loss and severe deformity of the cranium.

\section{Conclusion}

FD is a benign congenital condition that results in replacement of normal bone architecture with fibro-osseous tissue. The condition can be monostotic or polyostotic and can be in association with endocrine abnormalities in the context of McCune-Albright Syndrome and Mazabraud Syndrome. Disruption of bone structure predisposes these patients to deformity and fracture, but the overall prognosis is good. Radiologists should be aware of the potential for some of these lesions to rarely undergo malignant transformation in polyostotic FD cases. The characteristic ground-glass appearance on CT is pathognomonic. In this patient the MRI perfusion images were useful in confirming the avascular nature of the lesion and in helping to exclude potential vascular lesions such as Paget's disease, metastatic disease and invasive meningioma. Most patients can be managed conservatively, with surgical options reserved for those with extensive deformity, fracture, or malignant transformation. In the patient reported here, surgical intervention was necessitated by the severe mass-effect on the ocular structures from the expansile bony lesion with resultant proptosis, eye pain, redness and potential for optic nerve compression.

\section{Abbreviations}

FD - Fibrous dysplasia, CF-FD - Craniofacial Fibrous dysplasia, CT Computerized Tomography, MRI - Magnetic Resonance Imaging. PET Positron Emission Tomography.

\section{Conflict Of Interest: None.}

Funding: None.

References 
1. Riddle MD, Bui MM. (2013) Fibrous Dysplasia. Archives of pathology and laboratory medicine. 137(1):134-138.

2. Casselman J, Jonge ID, Neyt L, Clercq CD, D'hont G. (1993) MRI in Craniofacial Fibrous Dysplasia Neuroradiology. 35:234-237.

3. Kushchayeva YS, Kushchayev SV, Glushko TY, et al. (2018) Fibrous Dysplasia For Radiologists: Beyond Ground Glass Bone Matrix. Insights into imaging. 9(6):1035-1056.

4. Macdonald-Jankowski D. (2009) Fibrous Dysplasia: a Systematic Review. Dentomaxillo facial radiology. 38:196215.

5. Shah ZK, Peh Wcg, Koh WL, Shek Twh. (2005) Magnetic Resonance Imaging Appearances of Fibrous Dysplasia. The british journal of radiology. 78:1104-1115.

6. Kransdorf MJ, Moser RP, Gilkey FW. (1990) Fibrous Dysplasia. Radiographics. 10(3):519-537.

7. Brown EW, Megerian CA, Mckenna MJ, Weber A. (1994) Fibrous Dysplasia of the Temporal Bone: Imaging Findings. American journal of roentgenology. 164(3):679-682.

8. Kumar R, Madewell JE, Lindell MM, Swischuk LE. (1990) Fibrous Lesions of Bones. Radiographics. 10:237-256.

9. Macdonald DS, Li TK. (2009) fibrous Dysplasia in a Hong Kong Community: The Clinical and Radiological Features and Outcomes of Treatment. Oral and Maxillofacial Radiology. 38:63-72.

10. Bousson V, Rey-Jouvin C, Laredo J-D, et al. (2014) Fibrous Dysplasia And Mccune-Albright Syndrome: Imaging for Positive and Differential Diagnoses, Prognosis, and Follow-Up Guidelines. European Journal of Radiology. 83(10):1828-1842.
11. Fitzpatrick KA, Taljanovic MS, Speer DP, et al. (2004) Imaging Findings of Fibrous Dysplasia with Histopathologic and Intraoperative Correlation. American Journal of Roentgenology. 182:1389-1398.

12. Jee WH, Choi K-H, Choe B-Y, Park J-M, Shinn K-S. (1996) Fibrous Dysplasia: MR Imaging Characteristics with Radiopathologic Correlation. American Journal of Roentgenology. 167(6):1523-1527.

13. Daffner RH, Kirks DR, John A. Gehweiler J, Heaston DK. (1982) Computed Tomography of Fibrous Dysplasia. American Journal of Roentgenology. 139:943-948.

14. Osborn AG, Salzman KL, Jhaveri MD. (2016) Diagnostic Imaging Brain. In: 3 ed. Philadelphia, pa: Elsevier. 376-379.

15. Slootweg PJ, El-Naggar AK. (2018) World Health Organization 4th Edition of Head and Neck Tumor Classification: Insight into the Consequential Modifications. Virchows Arch. 472(3):311-313.

16. Mills SE, Greenson JK, Hornick JL, Longacre TA, Reuter VE. (2015) Sternberg's Diagnostic Surgical Pathology. Walters Kluwer Health.

17. Nelson BL, Phillips BJ. (2019) Benign Fibro-Osseous Lesions of the Head and Neck. Head neck pathol. 13(3):466-475.

18. Pereira TDSF, Gomes CC, Brennan PA, Fonseca FP, Gomez RS. (2019) Fibrous Dysplasia of the Jaws: Integrating Molecular Pathogenesis with Clinical, Radiological, and Histopathological Features. J Oral Pathol Med. 48(1):3-9.

19. Dicaprio MR, Enneking WF. (2005) Fibrous Dysplasia. Pathophysiology, Evaluation, and Treatment. The Journal of Bone and Joint Surgery. 87:1848-1864.

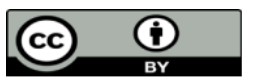

This work is licensed under Creative Commons Attribution 4.0 License

To Submit Your Article Click Here: Submit Manuscript

DOI: $10.31579 / 2690-4861 / 144$
Ready to submit your research? Choose Auctores and benefit from:

* fast, convenient online submission
* rigorous peer review by experienced research in your field
*apid publication on acceptance
* authors retain copyrights
* imique DOI for all articles
immediate, unrestricted online access

At Auctores, research is always in progress.

Learn more www.auctoresonline.org/journals/international-journal-ofclinical-case-reports-and-reviews- 